\title{
Identification of the collision kernel in the linear Boltzmann equation by a finite number of measurements on the boundary
}

\author{
ROLCI CIPOLATTI \\ Departamento de Métodos Matemáticos, Instituto de Matemática \\ Universidade Federal do Rio de Janeiro, Cx. Postal 68530, 21945-970 Rio de Janeiro, RJ, Brasil \\ E-mail: cipolatti@im.ufrj.br
}

\begin{abstract}
In this paper we consider the inverse problem of recovering the collision kernel for the time dependent linear Boltzmann equation via a finite number of boundary measurements. We prove that this kernel can be uniquely determined by at most $k$ measurements, provided that it belongs to a finite $k$-dimensional vector space.
\end{abstract}

Mathematical subject classification: 35R30, 83D75.

Key words: inverse problem, linear Boltzmann equation, albedo operator, boundary measurements.

\section{Introduction}

In this paper we consider an inverse problem for the linear Boltzmann equation

$$
\partial_{t} u+\omega \cdot \nabla_{x} u+q u=q K_{\kappa}[u] \text { in }(0, T) \times \mathbb{S} \times \Omega,
$$

where $T>0, \Omega$ is a smooth bounded convex domain of $\mathbb{R}^{N}, N \geq 2, \mathbb{S}$ denotes the unit sphere of $\mathbb{R}^{N}, q \in L^{\infty}(\Omega)$ and $K_{\kappa}$ is the integral operator with kernel $\kappa\left(x, \omega^{\prime}, \omega\right)$ defined by

$$
K_{\kappa}[u](t, \omega, x)=\int_{\mathbb{S}} \kappa\left(x, \omega^{\prime}, \omega\right) u\left(t, \omega^{\prime}, x\right) d \omega^{\prime} .
$$

\#691/06. Received: 01/IX/06. Accepted: 01/X/06. 
In applications, the equation (1.1) describes the dynamics of a monokinetic flow of particles in a body $\Omega$ under the assumption that the interaction between them is negligible (which allows us to discard nonlinear terms). For instance, in the case of a low-density flux of neutrons (see [7], [10]), $q \geq 0$ is the total extinction coefficient and the collision kernel $\kappa$ is given by

$$
\kappa\left(x, \omega^{\prime}, \omega\right)=c(x) h\left(x, \omega^{\prime} \cdot \omega\right),
$$

where $c$ corresponds to the within-group scattering probability and $h$ describes the anisotropy of the scattering process. In this model, $q(x) u(t, \omega, x)$ describes the loss of particles at $x$ in the direction $\omega$ at time $t$ due to absorption or scattering and $q(x) K_{\kappa}[u](t, \omega, x)$ represents the production of particles at $x$ in the direction $\omega$ from those coming from directions $\omega^{\prime}$.

Our focus here is the inverse problem of recovery the coefficients in (1.1) via boundary measurements. More precisely, we are interested to recover $q$ and $\kappa$ by giving the incoming flux of particles on the boundary and measuring the outgoing one. Since these operations are described mathematically by the albedo operator

$$
\mathcal{A}_{q, \kappa}: L^{1}\left(0, T ; L^{1}\left(\Sigma^{-} ; d \xi\right)\right) \longrightarrow L^{1}\left(0, T ; L^{1}\left(\Sigma^{+} ; d \xi\right)\right)
$$

(the spaces will be precised below), a general mathematical question concerning this inverse problem is to know if the knowledge of $\mathcal{A}_{q, \kappa}$ uniquely determines $q$, $\kappa$, i.e., if the map $(q, \kappa) \mapsto \mathcal{A}_{q, \kappa}$ is invertible.

Taking into account the applications, we have to precise this question. A first one is to know if the knowledge of $\mathcal{A}_{q, \kappa}[f]$ for all $f$ determines $(q, \kappa)$ (infinitely many measurements); a second one is to know if the knowledge of $\mathcal{A}_{q, \kappa}\left[f_{j}\right]$, for $j=1,2, \ldots, k$, determines $(q, \kappa)$ (finite number of measurements).

There is a wide bibliography devoted to the first problem. We specially mention the general results obtained by Choulli and Stefanov [4]: they show that $q$ and $\kappa$ are uniquely determined by the albedo operator (see also [9]). We also mention the stability results obtained by Cipolatti, Motta and Roberty (see [5] and the references therein).

There is also a lot of papers concerning the stationary case (see for instance those by V.G. Romanov [11], [12], P. Stefanov and G. Uhlmann [13], Tamasan [14], J.N. Wang [15], and also the references therein). 
In this work we focus on the second question, concerning the recovery by a finite number of measurements. This may be interesting from the numerical point of view (finite element methods, for instance). Assuming that $\kappa\left(t, \omega^{\prime}, \omega\right)=$ $c(x) h\left(\omega^{\prime}, \omega\right)$, we prove that $c$ can be uniquely determined by at most $k$ measurements, provided that $c$ belongs to a finite $k$-dimensional vector space of $C(\bar{\Omega})$. More precisely:

Theorem 1.1. Let $\Omega \subset \mathbb{R}^{N}$ be a bounded convex domain of class $C^{1}, T>$ $\operatorname{diam}(\Omega)$ and $\mathcal{X}:=\operatorname{span}\left\{\rho_{1}, \rho_{2}, \ldots, \rho_{k}\right\}$, where $\left\{\rho_{1}, \rho_{2}, \ldots, \rho_{k}\right\}$ is a linearly independent subset of $C(\bar{\Omega})$. We assume that $c \in \mathcal{X}$ and $\kappa\left(x, \omega^{\prime}, \omega\right)=$ $c(x) h\left(\omega^{\prime}, \omega\right)$, where $h \in C(\mathbb{S} \times \mathbb{S})$ satisfies $h(\omega, \omega) \neq 0$ for every $\omega \in \mathbb{S}$. Then, there exist $f_{1}, \ldots, f_{k} \in C_{0}\left((0, T) \times \Sigma^{-}\right)$and $\widetilde{\omega}_{1}, \ldots, \widetilde{\omega}_{k} \in \mathbb{S}$ that determine $\kappa$ uniquely.

The proof of Theorem 1.1 is based on the construction of highly oscillatory solutions (à la Calderón [1]) introduced in [5] and some arguments already used by the author in [6]. In fact, we consider solutions of the form

$$
u_{j}(t, \omega, x)=\chi_{s}\left(\widetilde{\omega}_{j}, \omega\right) \phi_{j}(x-t \omega) \mathrm{e}^{-\int_{0}^{t} \tilde{q}(x-\tau \omega) d \tau} \mathrm{e}^{\mathrm{i} \lambda(t-x \cdot \omega)}+R_{\lambda, s}(t, \omega, x),
$$

where $\chi_{s}$ converges (as $s \rightarrow 1$ ) to $\delta_{\tilde{\omega}_{j}}$, the spherical atomic measure concentrated on $\widetilde{\omega}_{j}$ and $R_{\lambda, s}$ vanishes as $\lambda \rightarrow \infty$. Therefore, by choosing $\widetilde{\omega}_{j}$ and $\phi_{j}$ conveniently, we obtain the result.

We organize the paper as follows: in Section 2 we recall the standard functional framework in which the Cauchy problem for (1.1) is well posed in the sense of the semigroup theory and the albedo operator is defined; in Section 3, we introduce the highly oscillatory functions that will be used, in Section 4, to prove Theorem 1.1.

\section{Notation and functional framework}

In this section we introduce the notation and we recall some well known results on the Transport Operator and the semigroup it generates in the Neutronic Function Spaces (see [5] and the references therein for the proofs).

Let $\Omega \subset \mathbb{R}^{N}(N \geq 2)$ be a convex and bounded domain of class $C^{1}$ and $\mathbb{S}$ the unit sphere of $\mathbb{R}^{N}$. We denote by $Q:=\mathbb{S} \times \Omega$ and $\Sigma$ its boundary, i.e., 
$\Sigma:=\mathbb{S} \times \partial \Omega$. For $p \in[1,+\infty)$ we consider the space $L^{p}(Q)$ with the usual norm

$$
\|u\|_{L^{p}(Q)}:=\left(\int_{Q}|u(\omega, x)|^{p} d x d \omega\right)^{1 / p},
$$

where $d \omega$ denotes the surface measure on $\mathbb{S}$ associated to the Lebesgue measure in $\mathbb{R}^{N-1}$.

For each $u \in L^{p}(Q)$ we define $A_{0} u$ by

$$
\left(A_{0} u\right)(\omega, x):=\omega \cdot \nabla_{x} u(\omega, x)=\sum_{k=1}^{N} \omega_{k} \frac{\partial u}{\partial x_{k}}(\omega, x), \quad \omega=\left(\omega_{1}, \ldots, \omega_{N}\right)
$$

where the derivatives are taken in the sense of distributions in $\Omega$.

One checks easily that setting $\mathcal{W}_{p}:=\left\{u \in L^{p}(Q) ; A_{0} u \in L^{p}(Q)\right\}$, the operator $\left(A_{0}, \mathcal{W}_{p}\right)$ is a closed densely defined operator and $\mathcal{W}_{p}$ with the graph norm is a Banach space.

For every $\sigma \in \partial \Omega$, we denote $v(\sigma)$ the unit outward normal at $\sigma \in \partial \Omega$ and we consider the sets (respectively, the incoming and outgoing boundaries)

$$
\Sigma^{ \pm}:=\{(\omega, \sigma) \in \mathbb{S} \times \partial \Omega ; \pm \omega \cdot v(\sigma)>0\} .
$$

In order to well define the albedo operator as a trace operator on the outgoing boundary, we consider $L^{p}\left(\Sigma^{ \pm} ; d \xi\right)$, where $d \xi:=|\omega \cdot v(\sigma)| d \sigma d \omega$, and we introduce the spaces

$$
\widetilde{\mathcal{W}}_{p}^{ \pm}:=\left\{u \in \mathcal{W}_{p} ; u_{\left.\right|_{\Sigma^{ \pm}}} \in L^{p}\left(\Sigma^{ \pm} ; \xi\right)\right\}
$$

which are Banach spaces if equipped with the norms

$$
\|u\|_{\widetilde{W}_{p}^{ \pm}}:=\left(\|u\|_{\mathcal{W}_{p}}^{p}+\int_{\Sigma^{ \pm}}|\omega \cdot v(\sigma) \| u(\omega, \sigma)|^{p} d \sigma d \omega\right)^{1 / p} .
$$

The next two lemmas concern the continuity and surjectivity of the trace operators (see [2], [3] and [5]):

$$
\gamma_{ \pm}: \widetilde{W}_{p}^{ \pm} \rightarrow L^{p}\left(\Sigma^{\mp} ; d \xi\right), \quad \gamma_{ \pm}(u):=u_{\left.\right|_{\Sigma \mp}} .
$$


Lemma 2.2. Let $1 \leq p<+\infty$. Then there exists $C>0$ (depending only on p) such that

$$
\int_{\Sigma^{\mp}}\left|\omega \cdot v(\sigma)\left\|\left.u(\omega, \sigma)\right|^{p} d \sigma d \omega \leq C\right\| u \|_{\widetilde{\mathcal{W}}_{p}^{ \pm}}^{p}, \quad \forall u \in \widetilde{\mathcal{W}}_{p}^{ \pm} .\right.
$$

Moreover, if $p>1$ and $1 / p+1 / p^{\prime}=1$, we have the Gauss identity

$$
\int_{Q} \operatorname{div}_{x}(u v \omega) d x d \omega=\int_{\Sigma} \omega \cdot v(\sigma) u(\omega, \sigma) v(\omega, \sigma) d \sigma d \omega,
$$

for all $u \in \widetilde{\mathcal{W}}_{p}^{ \pm}$and $v \in \widetilde{\mathcal{W}}_{p^{\prime}}^{ \pm}$

As an immediate consequence of Lemma 2.1, we can introduce the space

$$
\widetilde{\mathcal{W}}_{p}:=\left\{f \in \mathcal{W}_{p} ; \int_{\Sigma}|\omega \cdot v(\sigma)||f(\omega, \sigma)|^{p} d \omega d \sigma<+\infty\right\}
$$

an we have that $\widetilde{\mathcal{W}}_{p}^{+}=\widetilde{\mathcal{W}}_{p}^{-}=\widetilde{\mathcal{W}}_{p}$ with equivalent norms.

Lemma 2.2. The trace operators $\gamma_{ \pm}$are surjective from $\widetilde{W}_{p}^{ \pm}$onto $L^{p}\left(\Sigma^{\mp} ; d \xi\right)$. More precisely, for each $f \in L^{p}\left(\Sigma^{\mp} ; d \xi\right)$, there exists $h \in \widetilde{\mathcal{W}}_{p}^{ \pm}$such that $\gamma_{ \pm}(h)=f$ and

$$
\|h\| \widetilde{W}_{p}^{ \pm} \leq C\|f\|_{L^{p}\left(\Sigma^{\mp}, d \xi\right)},
$$

where $C>0$ is independent of $f$.

We consider the operator $A: D(A) \rightarrow L^{p}(Q)$, defined by $(A u)(\omega, x):=$ $\omega \cdot \nabla u(\omega, x)$, with $D(A):=\left\{u \in \widetilde{W}_{p} ; \gamma_{-}(u)=0\right\}$.

Theorem 2.3. The operator $A$ is $m$-accretive in $L^{p}(Q)$, for $p \in[1,+\infty)$.

Corollary 2.4. Let $f \in L^{p}(Q), p \in[1,+\infty)$ and assume that $u \in D(A)$ is a solution of $u+A u=f$. If $f \geq 0$ a.e. in $Q$, then $u \geq 0$ a.e. in $Q$. In particular, it follows that

$$
\|u\|_{L^{1}(Q)} \leq\|f\|_{L^{1}(Q)} .
$$

It follows from Theorem 2.3 and Corollary 2.4 that the operator $A$ generates a positive semigroup $\left\{U_{0}(t)\right\}_{t \geq 0}$ of contractions acting on $L^{p}(Q)$. 
Let $q \in L^{\infty}(\Omega)$ and $\kappa: \Omega \times \mathbb{S} \times \mathbb{S} \rightarrow \mathbb{R}$ be a real measurable function satisfying

$$
\left\{\begin{array}{l}
\int_{\mathbb{S}}\left|\kappa\left(x, \omega^{\prime}, \omega\right)\right| d \omega^{\prime} \leq M_{1} \text { a.e. } \Omega \times \mathbb{S} \\
\int_{\mathbb{S}}\left|\kappa\left(x, \omega^{\prime}, \omega\right)\right| d \omega \leq M_{2} \text { a.e. } \Omega \times \mathbb{S} .
\end{array}\right.
$$

Associated to these functions, we define the following continous operators:

1) $B \in \mathcal{L}\left(L^{p}(Q), L^{p}(Q)\right)$ defined by $B[u](\omega, x):=q(x) u(\omega, x)$,

2) $K_{\kappa}[u](\omega, x):=\int_{\mathbb{S}} \kappa\left(x, \omega^{\prime}, \omega\right) u\left(\omega^{\prime}, x\right) d \omega^{\prime}$.

It follows from (2.4) that $K_{\kappa} \in \mathcal{L}\left(L^{p}(Q), L^{p}(Q)\right) \forall p \in[1,+\infty)$ and (see [7])

$$
\left\|K_{\kappa}[u]\right\|_{L^{p}(Q)} \leq M_{1}^{1 / p^{\prime}} M_{2}^{1 / p}\|u\|_{L^{p}(Q)} \leq \max \left\{M_{1}, M_{2}\right\}\|u\|_{L^{p}(Q)} .
$$

The operator $A+B-K_{\kappa}: D(A) \rightarrow L^{p}(Q)$ generates a $c_{0}$-semigroup $\{U(t)\}_{t \geq 0}$ on $L^{p}(Q)$ satisfying

$$
\|U(t)\|_{\mathcal{L}} \leq \mathrm{e}^{C t}, \quad C:=\left\|q^{-}\right\|_{\infty}+M_{2} .
$$

We consider the initial-boundary value problem for the linear Boltzmann equation

$$
\left\{\begin{array}{l}
\partial_{t} u(t, \omega, x)+\omega \cdot \nabla u(t, \omega, x)+q(x) u(t, \omega, x)=q K_{\kappa}[u](t, \omega, x) \\
u(0, \omega, x)=0, \quad(\omega, x) \in \mathbb{S} \times \Omega \\
u(t, \omega, \sigma)=f(t, \omega, \sigma), \quad(\omega, \sigma) \in \Sigma^{-}, \quad t \in(0, T),
\end{array}\right.
$$

where $q \in L^{\infty}(\Omega), K_{\kappa}[u]$ is defined by (1.2) with $\kappa$ satisfying (2.4).

By the previous results, it follows that, for $f \in L^{p}\left(0, T ; L^{p}\left(\Sigma^{-}, d \xi\right)\right), p \in$ $[1,+\infty)$, there exists a unique solution $u \in C\left([0, T] ; \widetilde{\mathcal{W}}_{p}\right) \cap C^{1}\left([0, T] ; L^{p}(Q)\right)$ of (2.7). This solution $u$ allows us to define the albedo operator

$$
\begin{gathered}
\mathcal{A}_{q, \kappa}: L^{p}\left(0, T ; L^{p}\left(\Sigma^{-}, d \xi\right)\right) \rightarrow L^{p}\left(0, T ; L^{p}\left(\Sigma^{+}, d \xi\right)\right) \\
\mathcal{A}_{q, \kappa}[f](t, \omega, \sigma):=u(t, \omega, \sigma), \quad(\omega, \sigma) \in \Sigma^{+} .
\end{gathered}
$$


As a consequence of Lemmas 2.1 and 2.2, $\mathcal{A}_{q, \kappa}$ is a linear and bounded operator.

We also consider the following backward-boundary value problem, called the adjoint problem of (2.7):

$$
\left\{\begin{array}{l}
\partial_{t} u^{*}(t, \omega, x)+\omega \cdot \nabla u^{*}(t, \omega, x)-q(x) u^{*}(t, \omega, x)=-q K_{\kappa}^{*}\left[u^{*}\right](t, \omega, x) \\
u^{*}(T, \omega, x)=0, \quad(\omega, x) \in \mathbb{S} \times \Omega \\
u^{*}(t, \omega, \sigma)=f^{*}(t, \omega, \sigma), \quad(\omega, \sigma) \in \Sigma^{+}, t \in(0, T),
\end{array}\right.
$$

where $f^{*} \in L^{p^{\prime}}\left(0, T ; L^{p^{\prime}}\left(\Sigma^{+}, d \xi\right)\right), p^{\prime} \in[1,+\infty)$,

$$
K_{\kappa}^{*}\left[u^{*}\right]\left(t, \omega^{\prime}, x\right):=\int_{\mathbb{S}} \kappa\left(x, \omega^{\prime}, \omega\right) u^{*}(t, \omega, x) d \omega
$$

with the corresponding albedo operator $\mathcal{A}_{q, \kappa}^{*}$

$$
\begin{gathered}
\mathcal{A}_{q, \kappa}^{*}: L^{p^{\prime}}\left(0, T ; L^{p^{\prime}}\left(\Sigma^{+}, d \xi\right)\right) \rightarrow L^{p^{\prime}}\left(0, T ; L^{p^{\prime}}\left(\Sigma^{-}, d \xi\right)\right) \\
\mathcal{A}_{q, \kappa}^{*}\left[f^{*}\right](t, \omega, \sigma):=u^{*}(t, \omega, \sigma), \quad(\omega, \sigma) \in \Sigma^{-} .
\end{gathered}
$$

The operators $\mathcal{A}_{q, \kappa}$ and $\mathcal{A}_{q, \kappa}^{*}$ satisfy the following property:

\section{Lemma 2.5. Let}

$$
f \in L^{p}\left(0, T ; L^{p}\left(\Sigma^{-} ; d \xi\right)\right) \quad \text { and } \quad f^{*} \in L^{p^{\prime}}\left(0, T ; L^{p^{\prime}}\left(\Sigma^{+} ; d \xi\right)\right) \text {, }
$$

where $p, p^{\prime} \in(1,+\infty)$ are such that $1 / p+1 / p^{\prime}=1$. Then, we have

$$
\begin{aligned}
& \int_{0}^{T} \int_{\Sigma^{-}}(\omega \cdot v(\sigma)) f(t, \omega, \sigma) \mathcal{A}_{q, \kappa}^{*}\left[f^{*}\right](t, \omega, \sigma) d \sigma d \omega d t \\
& \quad=-\int_{0}^{T} \int_{\Sigma^{+}}(\omega \cdot v(\sigma)) f^{*}(t, \omega, \sigma) \mathcal{A}_{q, \kappa}[f](t, \omega, \sigma) d \sigma d \omega d t .
\end{aligned}
$$

Proof. It is a direct consequence of Lemma 2.1. Let $u(t, \omega, x)$ the solution of (2.7) with boundary condition $f$ and $u^{*}(t, \omega, x)$ the solution of (2.8) with boundary $f^{*}$. We obtain the result by using (2.3), once the equation in (2.7) is multiplied by $u^{*}$ and integrated over $(0, T) \times Q$.

As a direct consequence of Lemma 2.5, we have: 
Lemma 2.6. Let $T>0, q_{1}, q_{2} \in L^{\infty}(\Omega)$ and $\kappa_{1}, \kappa_{2}$ satisfying (2.4). Assume that $u_{1}$ is the solution of (2.7) with coefficients $q_{1}, \kappa_{1}$ and satisfying the boundary condition $f \in L^{p}\left(0, T ; L^{p}\left(\Sigma^{-}, d \xi\right)\right), p \in(1,+\infty)$ and that $u_{2}^{*}$ is the solution of (2.8), with $q_{2}, \kappa_{2}$ and boundary condition $f^{*} \in L^{p^{\prime}}\left(0, T ; L^{p^{\prime}}\left(\Sigma^{+}, d \xi\right)\right)$, $1 / p+1 / p^{\prime}=1$. Then we have

$$
\begin{aligned}
\int_{0}^{T} & \int_{Q}\left(q_{2}(x)-q_{1}(x)\right) u_{1}(t, \omega, x) u_{2}^{*}(t, \omega, x) d x d \omega d t \\
& \quad-\int_{0}^{T} \int_{Q}\left(q_{2}(x) K_{\kappa_{2}}\left[u_{1}\right](t, \omega, x)-q_{1}(x) K_{\kappa_{1}}\left[u_{1}\right](t, \omega, x)\right) u_{2}^{*}(t, \omega, x) d x d \omega d t \\
& =\int_{0}^{T} \int_{\Sigma^{+}}(\omega \cdot v(\sigma))\left[\mathcal{A}_{q_{1}, \kappa_{1}}[f]-\mathcal{A}_{q_{2}, \kappa_{2}}[f]\right](t, \omega, \sigma) f^{*}(t, \omega, \sigma) d \sigma d \omega d t .
\end{aligned}
$$

\section{Highly oscillatory solutions}

In this section we prove some technical results related to special solutions of (2.7) and (2.8) that will be useful in the proof of Theorem 1.1. We denote by $\tilde{q}$ the zero extension of $q$ in the exterior of $\Omega$.

Proposition 3.1. Let $T>0, q_{1}, q_{2} \in L^{\infty}(\Omega)$, and $\kappa_{1}, \kappa_{2}$ satisfying (2.4). We consider $\psi_{1}, \psi_{2} \in C\left(\mathbb{S}, C_{0}^{\infty}\left(\mathbb{R}^{N}\right)\right)$ such that

$$
\operatorname{supp} \psi_{1}(\omega, \cdot) \cap \bar{\Omega}=\left(\operatorname{supp} \psi_{2}(\omega, \cdot)+T \omega\right) \cap \bar{\Omega}=\emptyset, \quad \forall \omega \in \mathbb{S} .
$$

Then, there exists $C_{0}>0$ such that, for each $\lambda>0$, there exist $R_{1, \lambda} \in$ $C\left([0, T] ; \widetilde{W}_{2}\right)$ and $R_{2, \lambda}^{*} \in C\left([0, T] ; \widetilde{\mathcal{W}}_{2}\right)$ satisfying

$$
\left\|R_{1, \lambda}\right\|_{C\left([0, T] ; L^{2}(Q)\right)} \leq C_{0}, \quad\left\|R_{2, \lambda}^{*}\right\|_{C\left([0, T] ; L^{2}(Q)\right)} \leq C_{0},
$$

for which the functions $u_{1}, u_{2}^{*}$ defined by

$$
\left\{\begin{array}{l}
u_{1}(t, \omega, x):=\psi_{1}(\omega, x-t \omega) e^{-\int_{0}^{t} \tilde{q}_{1}(x-s \omega) d s} e^{\mathrm{i} \lambda(t-\omega \cdot x)}+R_{1, \lambda}(t, \omega, x) \\
u_{2}^{*}(t, \omega, x):=\psi_{2}(\omega, x-t \omega) e^{\int_{0}^{t} \tilde{q}_{2}(x-s \omega) d s} e^{-\mathrm{i} \lambda(t-\omega \cdot x)}+R_{2, \lambda}^{*}(t, \omega, x)
\end{array}\right.
$$

are solutions of (2.7) with $(q, \kappa)=\left(q_{1}, \kappa_{1}\right)$ and $(2.8)$ with $(q, \kappa)=\left(q_{2}, \kappa_{2}\right)$ respectively. Moreover, if $\kappa_{j} \in L^{\infty}\left(\Omega ; L^{2}(\mathbb{S} \times \mathbb{S})\right)$, then we have

$$
\lim _{\lambda \rightarrow+\infty}\left\|R_{1, \lambda}\right\|_{C\left([0, T] ; L^{2}(Q)\right)}=\lim _{\lambda \rightarrow+\infty}\left\|R_{2, \lambda}^{*}\right\|_{C\left([0, T] L^{2}(Q)\right)}=0 .
$$


Proof. Let $u$ be the function

$$
u(t, \omega, x):=\psi_{1}(\omega, x-t \omega) \mathrm{e}^{-\int_{0}^{t} \tilde{q}_{1}(x-s \omega) d s} \mathrm{e}^{\mathrm{i} \lambda(t-\omega \cdot x)}+R(t, \omega, x) .
$$

By direct calculations, we easily verify that

$$
\begin{gathered}
\partial_{t} u+\omega \cdot \nabla u+q_{1} u-q_{1} K_{\kappa_{1}}[u] \\
=\partial_{t} R+\omega \cdot \nabla R+q_{1} R-q_{1} K_{\kappa_{1}}[R]-\mathrm{e}^{\mathrm{i} \lambda t} q_{1} Z_{1, \lambda},
\end{gathered}
$$

where

$$
Z_{1, \lambda}(t, \omega, x):=\int_{\mathbb{S}} \kappa_{1}\left(x, \omega^{\prime}, \omega\right) \psi_{1}\left(\omega^{\prime}, x-t \omega^{\prime}\right) \mathrm{e}^{-\int_{0}^{t} \tilde{q}_{1}\left(x-s \omega^{\prime}\right) d s} \mathrm{e}^{-\mathrm{i} \lambda \omega^{\prime} \cdot x} d \omega^{\prime} .
$$

From (2..6), there exists $R_{1, \lambda} \in C^{1}\left([0, T] ; L^{2}(Q)\right) \cap C([0, T] ; D(A))$ a unique solution of

$$
\left\{\begin{array}{l}
\partial_{t} R+\omega \cdot \nabla R+q_{1} R=q_{1} K_{\kappa_{1}}[R]+\mathrm{e}^{\mathrm{i} \lambda t} q_{1} Z_{1, \lambda}, \\
R(0, \omega, x)=0, \quad(\omega, x) \in S \times \Omega, \\
R(t, \omega, \sigma)=0, \quad(\omega, \sigma) \in \Sigma^{-},
\end{array}\right.
$$

and it follows from (3.1) that the function $u$ defined by (3.5) satisfies (2.7) with boundary condition

$$
f_{\lambda}(t, \omega, \sigma):=\psi_{1}(\omega, \sigma-t \omega) \mathrm{e}^{-\int_{0}^{t} \tilde{q}_{1}(\sigma-s \omega) d s} \mathrm{e}^{\mathrm{i} \lambda(t-\omega \cdot \sigma)}, \quad(\omega, \sigma) \in \Sigma^{-} .
$$

Multiplying both sides of the equation in (3.7) by the complex conjugate of $R$, integrating it over $Q$ and taking its real part, we get, from Lemma 2.1,

$$
\begin{gathered}
\frac{1}{2} \frac{d}{d t} \int_{Q}|R(t)|^{2} d \omega d x+\frac{1}{2} \int_{\Sigma^{+}} \omega \cdot v(\sigma)|R(t)|^{2} d \omega d \sigma+\int_{Q} q_{1}|R(t)|^{2} d \omega d x \\
-\Re \int_{Q} q_{1} K_{\kappa_{1}}[R](t) \overline{R(t)} d \omega d x=\Re\left[\mathrm{e}^{\mathrm{i} \lambda t} \int_{Q} q_{1} Z_{1, \lambda}(t) \overline{R(t)} d \omega d x\right] .
\end{gathered}
$$

It follows from the Cauchy-Schwarz inequality and (2.5) that

$$
\int_{Q}\left|K_{\kappa_{1}}[R(t)]\right||R(t)| d x d \omega \leq C_{1}\|R(t)\|_{L^{2}(Q)}^{2},
$$

where $C_{1}:=\max \left\{M_{1}, M_{2}\right\}$. Therefore, we obtain

$$
\frac{d}{d t}\|R(t)\|_{L^{2}(Q)}^{2} \leq C_{2}\left\|q_{1}\right\|_{\infty}\|R(t)\|_{L^{2}(Q)}^{2}+\left\|q_{1}\right\|_{\infty}\left\|Z_{1, \lambda}(t)\right\|_{L^{2}(Q)}^{2},
$$


where $C_{2}$ : $=3+2 C_{1}$. Since $R(0)=0$, we get, by integrating this last inequality on $[0, t]$,

$$
\begin{aligned}
\|R(t)\|_{L^{2}(Q)}^{2} & \leq\left\|q_{1}\right\|_{\infty} \mathrm{e}^{\left\|q_{1}\right\|_{\infty} T C_{2}} \int_{0}^{t}\left\|Z_{1, \lambda}(\tau)\right\|_{L^{2}(Q)}^{2} d \tau \quad, \forall t \in[0, T] . \\
& \leq\left\|q_{1}\right\|_{\infty} \mathrm{e}^{\left\|q_{1}\right\|_{\infty} T C_{2}}\left\|Z_{1, \lambda}\right\|_{L^{2}((0, T) \times Q)}^{2}
\end{aligned}
$$

The first inequality in (3.2) follows easily because

$$
\left|Z_{1, \lambda}(t, \omega, x)\right| \leq\left\|\psi_{1}\right\|_{\infty} \mathrm{e}^{\left\|q_{1}\right\|_{\infty} T} M_{1}
$$

and, as the same arguments hold for $u_{2}^{*}$ and $R_{2, \lambda}^{*}$, we also obtain the second inequality.

We assume now $\kappa \in L^{\infty}\left(\Omega ; L^{2}(\mathbb{S} \times \mathbb{S})\right)$. For each $x \in \mathbb{R}^{N}$, the map $\omega^{\prime} \mapsto$ $\exp \left(\mathrm{i} \lambda \omega^{\prime} \cdot x\right)$ converges weakly to zero in $L^{2}(\mathbb{S})$ when $\lambda \rightarrow+\infty$ and the integral operator with kernel $\kappa(x, \cdot, \cdot)$ is compact in $L^{2}(\mathbb{S})$. So, we obtain from (3.6),

$$
\lim _{\lambda \rightarrow+\infty}\left\|Z_{1, \lambda}(t, \cdot, x)\right\|_{L^{2}(\mathbb{S})}=0 \quad \text { a.e. in } \quad[0, T] \times \Omega .
$$

Moreover, $\left\|Z_{1, \lambda}(t, \cdot, x)\right\|_{L^{2}(\mathbb{S})} \leq C$, where $C>0$ is a constant that does not depend on $\lambda$. The Lebesgue's Dominated Convergence Theorem implies that

$$
\lim _{\lambda \rightarrow+\infty}\left\|Z_{1, \lambda}\right\|_{L^{2}([0, T] \times Q)}=0 .
$$

From (3.9) and (3.8) we obtain (3.4), and our proof is complete.

Corollary 3.2. Under the hypothesis of Proposition 3.1, if $q_{1}, q_{2} \in C(\bar{\Omega})$ and $\kappa_{1}, \kappa_{2} \in L^{\infty}(\Omega ; C(\mathbb{S} \times \mathbb{S}))$, we have, for every $\omega \in \mathbb{S}$,

$$
\lim _{\lambda \rightarrow+\infty}\left\|R_{1, \lambda}(\cdot, \omega, \cdot)\right\|_{C\left([0, T] ; L^{2}(\Omega)\right)}=\lim _{\lambda \rightarrow+\infty}\left\|R_{2, \lambda}^{*}(\cdot, \omega, \cdot)\right\|_{C\left([0, T] ; L^{2}(\Omega)\right)}=0 .
$$

Proof. By multiplying both sides of the equation in (3.7) by the complex conjugate of $R(t, \omega, x)$, integrating it over $\Omega$, taking its real part and applying the Hölder inequality, we get

$$
\begin{gathered}
\frac{d}{d t}\|R(t, \omega)\|_{L^{2}(\Omega)}^{2} \leq 4\left\|q_{1}\right\|_{\infty}\|R(t, \omega)\|_{L^{2}(\Omega)}^{2} \\
+\left\|q_{1}\right\|_{\infty}\left(\left\|K_{\kappa_{1}}[R](t, \omega)\right\|_{L^{2}(\Omega)}^{2}+\left\|Z_{1, \lambda}(t, \omega)\right\|_{L^{2}(\Omega)}^{2}\right) .
\end{gathered}
$$




\section{Since}

$$
\begin{aligned}
& \left|K_{\kappa_{1}}[R](t, \omega, x)\right| \leq \int_{\mathbb{S}}\left|\kappa_{1}\left(x, \omega^{\prime}, \omega\right)\right|\left|R\left(t, \omega^{\prime}, x\right)\right| d \omega^{\prime} \\
& \quad \leq\left(\int_{\mathbb{S}}\left|\kappa_{1}\left(x, \omega^{\prime}, \omega\right)\right| d \omega^{\prime}\right)^{1 / 2}\left(\int_{\mathbb{S}}\left|\kappa_{1}\left(x, \omega^{\prime}, \omega\right) \| R\left(t, \omega^{\prime}, x\right)\right|^{2} d \omega^{\prime}\right)^{1 / 2} \\
& \quad \leq M_{1}^{1 / 2}\left\|\kappa_{1}\right\|_{\infty}^{1 / 2}\left(\int_{\mathbb{S}}\left|R\left(t, \omega^{\prime}, x\right)\right|^{2} d \omega^{\prime}\right)^{1 / 2},
\end{aligned}
$$

we obtain

$$
\left\|K_{\kappa_{1}}[R](t, \omega)\right\|_{L^{2}(\Omega)}^{2} \leq M_{1}\left\|\kappa_{1}\right\|_{\infty}\|R(t)\|_{L^{2}(Q)}^{2} .
$$

From (3.8), (3.10) and (3.11) we have

$$
\begin{aligned}
& \quad \frac{d}{d t}\|R(t, \omega)\|_{L^{2}(\Omega)}^{2} \leq 4\left\|q_{1}\right\|_{\infty}\|R(t, \omega)\|_{L^{2}(\Omega)}^{2} \\
& +C\left(\left\|Z_{1, \lambda}\right\|_{L^{2}((0, T) \times Q)}^{2}+\left\|Z_{1, \lambda}(t, \omega)\right\|_{L^{2}(\Omega)}^{2}\right) .
\end{aligned}
$$

Now, integrating this last inequality on time, we get

$$
\begin{aligned}
\|R(t, \omega)\|_{L^{2}(\Omega)}^{2} & \leq C \mathrm{e}^{\left\|q_{1}\right\|_{\infty} T}\left(t\left\|Z_{1, \lambda}\right\|_{L^{2}((0, T) \times Q)}^{2}+\int_{0}^{t}\left\|Z_{1, \lambda}(\tau, \omega)\right\|_{L^{2}(\Omega)}^{2} d \tau\right) \\
& \leq C \mathrm{e}^{\left\|q_{1}\right\|_{\infty} T}\left(T\left\|Z_{1, \lambda}\right\|_{L^{2}((0, T) \times Q)}^{2}+\left\|Z_{1, \lambda}(\cdot, \omega, \cdot)\right\|_{L^{2}((0, T) \times \Omega)}^{2}\right) .
\end{aligned}
$$

From Proposition 3.1 we know that $\left\|Z_{1, \lambda}\right\|_{L^{2}((0, T) \times Q)} \rightarrow 0$ as $\lambda \rightarrow+\infty$. On the other hand, as the map $\omega^{\prime} \mapsto \mathrm{e}^{\mathrm{i} \omega^{\prime} \cdot x}$ converges weakly to zero in $L^{2}(\mathbb{S})$, we have from (3.6), for almost $x \in \Omega$,

$$
\lim _{\lambda \rightarrow \infty} Z_{1, \lambda}(t, \omega, x)=0, \quad \forall \omega \in S, \forall t \in[0, T]
$$

and the conclusion follows from the Lebesgue's Theorem.

Lemma 3.3. We assume that $q \in L^{\infty}(\Omega)$ and $\kappa$ satisfies (2.4). Let $S_{\lambda}^{*}$ be the solution of

$$
\left\{\begin{array}{l}
\partial_{t} S+\omega \cdot \nabla S-q S=-q K_{\kappa}^{*}[S]+q e^{-\mathrm{i} \lambda t} Z, \\
S(T, \omega, x)=0, \quad(\omega, x) \in \mathbb{S} \times \Omega, \\
S(t, \omega, \sigma)=0, \quad(\omega, \sigma) \in \Sigma^{+},
\end{array}\right.
$$


where $Z \in H^{1}\left(0, T ; L^{2}(Q)\right)$ such that $Z(T)=0$. Then we have

$$
\left\|S_{\lambda}^{*}\right\|_{C\left([0, T] ; L^{2}(Q)\right)} \leq C_{0} \quad \text { and } \quad \lim _{\lambda \rightarrow \infty}\left\|S_{\lambda}^{*}\right\|_{H^{-1}\left(0, T ; L^{2}(Q)\right)}=0,
$$

where $C_{0}$ is a constant independent of $\lambda$.

Proof. Multiplying both sides of the equation in (3.12) by the complex conjugate of $S_{\lambda}^{*}$, integrating it over $Q$ and taking its real part, we get

$$
\begin{gathered}
\frac{1}{2} \frac{d}{d t}\left\|S_{\lambda}^{*}(t)\right\|_{L^{2}(Q)}^{2}+\frac{1}{2} \int_{\Sigma^{-}}(\omega \cdot v(\sigma))\left|S_{\lambda}^{*}(t, \omega, \sigma)\right| d \omega d \sigma \geq-\|q\|_{\infty}\left\|S_{\lambda}^{*}(t)\right\|_{L^{2}(Q)}^{2} \\
-\|q\|_{\infty}\left\|K_{\kappa}^{*}[S](t)\right\|_{L^{2}(Q)}\left\|S_{\lambda}^{*}(t)\right\|_{L^{2}(Q)}-\|q\|_{\infty}\|Z(t)\|_{L^{2}(Q)}\left\|S_{\lambda}^{*}(t)\right\|_{L^{2}(Q)}
\end{gathered}
$$

Since

$$
\left\|K_{\kappa}^{*}[S](t)\right\|_{L^{2}(Q)} \leq \max \left\{M_{1}, M_{2}\right\}\left\|S_{\lambda}^{*}(t)\right\|_{L^{2}(Q)},
$$

we have

$$
\frac{d}{d t}\left\|S_{\lambda}^{*}(t)\right\|_{L^{2}(Q)}^{2} \geq-C_{2}\left\|S_{\lambda}^{*}(t)\right\|_{L^{2}(Q)}^{2}-\|q\|_{\infty}\|Z(t)\|_{L^{2}(Q)}^{2},
$$

where $C_{2}:=\left(3+2 \max \left\{M_{1}, M_{2}\right\}\right)\|q\|_{\infty}$. Integrating this last inequality on $[t, T]$ and taking into account that $S_{\lambda}^{*}(T)=0$, we obtain

$$
\begin{aligned}
\left\|S_{\lambda}^{*}(t)\right\|_{L^{2}(Q)}^{2} & \leq\|q\|_{\infty} \mathrm{e}^{C_{2} T} \int_{t}^{T}\|Z(\tau)\|_{L^{2}(Q)}^{2} d \tau \\
& \leq\|q\|_{\infty} \mathrm{e}^{C_{2} T}\|Z\|_{L^{2}\left(0, T ; L^{2}(Q)\right)}
\end{aligned}
$$

and the inequality in (3.13) follows easily.

We consider now

$$
\begin{aligned}
w_{\lambda}(t, \omega, x) & :=\int_{t}^{T} S_{\lambda}^{*}(\tau, \omega, x) d \tau, \\
h(t, \omega, x): & =\int_{t}^{T} \mathrm{e}^{-\mathrm{i} \lambda \tau} Z(\tau, \omega, x) d \tau .
\end{aligned}
$$

Then, it is easy to check that $w_{\lambda}$ satisfies

$$
\left\{\begin{array}{l}
\partial_{t} w+\omega \cdot \nabla w-q w=-q K_{\kappa}^{*}[w]+q h, \\
w(T, \omega, x)=0, \quad(\omega, x) \in \mathbb{S} \times \Omega, \\
w(t, \omega, \sigma)=0, \quad(\omega, \sigma) \in \Sigma^{+},
\end{array}\right.
$$


Multiplying both sides of the equation in (3.16) by the complex conjugate of $w_{\lambda}$, integrating it over $Q$, taking its real part and applying the Cauchy-Schwarz inequality, we get as before,

$$
\begin{aligned}
\left\|w_{\lambda}(t)\right\|_{L^{2}(Q)}^{2} & \leq\|q\|_{\infty} \mathrm{e}^{C_{2} T}\|h\|_{L^{2}\left(0, T ; L^{2}(Q)\right)}^{2} \\
& \leq\|q\|_{\infty} T^{2} \mathrm{e}^{C_{2} T}\|Z\|_{L^{2}\left(0, T ; L^{2}(Q)\right)}^{2} .
\end{aligned}
$$

As $S_{\lambda}^{*}=-\partial_{t} w_{\lambda}$, it follows from (3.14) and (3.17) that the set $\left\{w_{\lambda}\right\}$ is bounded in $C^{1}\left([0, T] ; L^{2}(Q)\right)$ and, in particular, is relatively compact in $C\left([0, T] ; L^{2}(Q)\right)$.

On the other hand, by integrating by parts the second integral in (3.15), it is easy to check that there exists $C>0$ (depending only on $T$ ) such that

$$
\|h\|_{L^{2}\left(0, T: L^{2}(Q)\right)} \leq \frac{C}{|\lambda|}\|Z\|_{H^{1}\left(0, T ; L^{2}(Q)\right)} .
$$

Hence, by (3.17), it follows that $\left\|w_{\lambda}\right\|_{C\left([0, T] ; L^{2}(Q)\right)} \rightarrow 0$ as $\lambda \rightarrow \infty$. Since the partial derivative in $t, \partial_{t}: C\left([0, T] ; L^{2}(Q)\right) \rightarrow H^{-1}\left(0, T ; L^{2}(Q)\right)$, is a continuous operator, there exists a constant $C_{3}>0$ such that

$$
\left\|S_{\lambda}^{*}\right\|_{H^{-1}\left(0, T ; L^{2}(Q)\right)}=\left\|\partial_{t} w_{\lambda}\right\|_{H^{-1}\left(0, T ; L^{2}(Q)\right)} \leq C_{2}\left\|w_{\lambda}\right\|_{C\left(0, T ; L^{2}(Q)\right)}
$$

and we have the conclusion.

\section{Recovery by a finite number of boundary measurements}

In this section we assume that $\left\{\rho_{1}, \rho_{2}, \ldots, \rho_{k}\right\}$ is a given linearly independent set of functions of $C(\bar{\Omega})$ and we denote $\mathcal{X}:=\operatorname{span}\left\{\rho_{1}, \rho_{2}, \ldots, \rho_{k}\right\}$. For each $\widetilde{\omega} \in \mathbb{S}$ we consider $P_{\tilde{\omega}}\left[\rho_{i}\right]$ the X-ray transform of $\rho_{i}$ in the direction $\widetilde{\omega}$, i.e.,

$$
P_{\tilde{\omega}}\left[\rho_{i}\right](x):=\int_{-\infty}^{\infty} \rho_{i}(x+t \widetilde{\omega}) d t
$$

and, for each $\varepsilon>0, \Omega_{\varepsilon}:=\left\{x \in \mathbb{R}^{N} \backslash \bar{\Omega} ; \operatorname{dist}(x, \Omega)<\varepsilon\right\}$.

The following Lemma, which the proof is given in [6], will be essential for the proof of Theorem 1.1: 
Lemma 4.1. For all $\varepsilon>0$, there exist $\widetilde{\omega}_{j} \in \mathbb{S}$ and $\phi_{j} \in C_{0}^{\infty}\left(\Omega_{\varepsilon}\right), j=$ $1, \ldots, k$, such that the matrix $A=\left(a_{i j}\right)$, with entries defined by

$$
a_{i j}:=\int_{\mathbb{R}^{N}} P_{\tilde{\omega}_{j}}\left[\rho_{i}\right](x) \phi_{j}^{2}(x) d x
$$

is invertible.

In order to prove Theorem 1.1, we define, for $0<r<1$, the function $\chi_{r}: \mathbb{S} \times$ $\mathbb{S} \rightarrow \mathbb{R}$ as $\chi_{r}(\widetilde{\omega}, \omega):=P(r \tilde{\omega}, \omega)$, where $P$ is the Poisson kernel for $B_{1}(0)$, i.e.,

$$
P(x, y):=\frac{1-|x|^{2}}{\alpha_{N}|x-y|^{N}} .
$$

From the well known properties of $P$ (see [8]), we have

$$
\begin{aligned}
& \int_{\mathbb{S}} \chi_{r}(\widetilde{\omega}, \omega) d \omega=1, \quad \forall r \in(0,1), \quad \forall \widetilde{\omega} \in \mathbb{S}, \\
& \lim _{r \rightarrow 1} \int_{\mathbb{S}} \chi_{r}(\widetilde{\omega}, \omega) \psi(\omega) d \omega=\psi(\widetilde{\omega}),
\end{aligned}
$$

where the above limit is taken in the topology of $L^{p}(\mathbb{S}), p \in[1,+\infty)$ and uniformly on $\mathbb{S}$ if $\psi \in C(\mathbb{S})$. We are now in position to prove our main result.

Proof of Theorem 1.1. Let $\varepsilon:=(T-\operatorname{diam}(\Omega)) / 2$. We assume that $q_{1}=$ $q_{2}=q$ and $\kappa_{i}\left(x, \omega^{\prime}, \omega\right)=c_{i}(x) h\left(\omega^{\prime}, \omega\right)$, where $c_{1}, c_{2} \in \mathcal{X}$. For $\widetilde{\omega} \in \mathbb{S}$, we define $\psi_{1}(\omega, x)=\chi_{s}(\tilde{\omega}, \omega) \phi(x)$ and $\psi_{2}(\omega, x)=\chi_{r}(\widetilde{\omega}, \omega) \phi(x)$, where $0<r, s<1$ and $\phi \in C_{0}^{\infty}\left(\Omega_{\varepsilon}\right)$. Then $\psi_{1}$ and $\psi_{2}$ satisfy the condition (3.1) and we may consider the solutions $u_{1}$ and $u_{2}^{*}$ defined by (3.3), i.e.,

$$
\begin{aligned}
& u_{1}(t, \omega, x):=\chi_{s}(\widetilde{\omega}, \omega) \phi(x-t \omega) \mathrm{e}^{-\int_{0}^{t} \tilde{q}(x-\tau \omega) d \tau} \mathrm{e}^{\mathrm{i} \lambda(t-x \cdot \omega)}+R_{1, \lambda, s}(t, \omega, x), \\
& u_{2}^{*}(t, \omega, x):=\chi_{r}(\widetilde{\omega}, \omega) \phi(x-t \omega) \mathrm{e}^{\int_{0}^{t} \tilde{q}(x-\tau \omega) d \tau} \mathrm{e}^{-\mathrm{i} \lambda(t-x \cdot \omega)}+R_{2, \lambda, r}^{*}(t, \omega, x),
\end{aligned}
$$

where $\lambda>0$ will be chosen a posteriori. We shall write

$$
\begin{aligned}
& \Phi_{\lambda}(t, \omega, x):=\phi(x-t \omega) \mathrm{e}^{-\int_{0}^{t} \tilde{q}(x-\tau \omega) d \tau} \mathrm{e}^{\mathrm{i} \lambda(t-x \cdot \omega)} \\
& \Psi_{\lambda}(t, \omega, x):=\phi(x-t \omega) \mathrm{e}^{\int_{0}^{t} \tilde{q}(x-\tau \omega) d \tau} \mathrm{e}^{-\mathrm{i} \lambda(t-x \cdot \omega)}
\end{aligned}
$$


in such a way that

$$
\begin{aligned}
& u_{1}(t, \omega, x)=\chi_{s}(\widetilde{\omega}, \omega) \Phi_{\lambda}(t, \omega, x)+R_{1, \lambda, s}(t, \omega, x), \\
& u_{2}^{*}(t, \omega, x)=\chi_{r}(\widetilde{\omega}, \omega) \Psi_{\lambda}(t, \omega, x)+R_{2, \lambda, r}^{*}(t, \omega, x) .
\end{aligned}
$$

Substituting $u_{1}$ and $u_{2}^{*}$ in the indentity given in Lemma 2.6, we have

$$
J(\lambda, r, s)=L(\lambda, r, s)
$$

where

$$
\begin{aligned}
& J(\lambda, r, s):=\int_{0}^{T} \int_{Q} q(x)\left(c_{1}(x)-c_{2}(x)\right) K_{h}\left[u_{1}\right](t, \omega, x) u_{2}^{*}(t, \omega, x) d x d \omega d t, \\
& L(\lambda, r, s):=\int_{0}^{T} \int_{\Sigma^{+}}(\omega \cdot v(\sigma))\left(\mathcal{A}_{1}\left[f_{\lambda, s}\right]-\mathcal{A}_{2}\left[f_{\lambda, s}\right]\right) f_{\lambda, r}^{*} d \sigma d \omega d t .
\end{aligned}
$$

In the above formulas, we are denoting $\mathcal{A}_{i}=\mathcal{A}_{c_{i}}, i=1,2$ and

$$
\begin{array}{ll}
f_{\lambda, s}(t, \omega, \sigma):=\chi_{s}(\widetilde{\omega}, \omega) \Phi_{\lambda}(t, \omega, \sigma), & (\omega, \sigma) \in \Sigma^{-} \\
f_{\lambda, r}^{*}(t, \omega, \sigma):=\chi_{r}(\widetilde{\omega}, \omega) \Psi_{\lambda}(t, \omega, \sigma), & (\omega, \sigma) \in \Sigma^{+} .
\end{array}
$$

In particular, it follows from the definition of the Albedo Operator and (4.3),

$$
\mathcal{A}_{1}\left[f_{\lambda, s}\right]-\mathcal{A}_{2}\left[f_{\lambda, s}\right]=R_{1, \lambda, s}-R_{2, \lambda, s}, \quad \text { on }(0, T) \times \Sigma^{+} \text {. }
$$

By denoting $\eta(x)=\widetilde{q}(x)\left(\widetilde{c}_{1}(x)-\widetilde{c}_{2}(x)\right)$ and by considering the special form of $u_{1}$ and $u_{2}^{*}$, we may write $J(\lambda, r, s)$ as $J=J_{1}+J_{2}+J_{3}+J_{4}$, where

$$
\begin{aligned}
J_{1}(\lambda, r, s):= & \int_{0}^{T} \int_{Q} \eta(x)\left[\int_{\mathbb{S}} h\left(\omega^{\prime}, \omega\right) \chi_{s}\left(\widetilde{\omega}, \omega^{\prime}\right) \Phi_{\lambda}\left(t, \omega^{\prime}, x\right) d \omega^{\prime}\right] \\
& \chi_{r}(\widetilde{\omega}, \omega) \Psi_{\lambda}(t, \omega, x) d x d \omega d t, \\
J_{2}(\lambda, r, s):= & \int_{0}^{T} \int_{Q} \eta(x)\left[\int_{\mathbb{S}} h\left(\omega^{\prime}, \omega\right) \chi_{s}\left(\widetilde{\omega}, \omega^{\prime}\right) \Phi_{\lambda}\left(t, \omega^{\prime}, x\right) d \omega^{\prime}\right] \\
& R_{2, \lambda, r}^{*}(t, \omega, x) d x d \omega d t, \\
J_{3}(\lambda, r, s):= & \int_{0}^{T} \int_{Q} \eta(x)\left[\int_{\mathbb{S}} h\left(\omega^{\prime}, \omega\right) R_{1, \lambda, s}\left(t, \omega^{\prime}, x\right) d \omega^{\prime}\right] \\
& \chi_{r}(\widetilde{\omega}, \omega) \Psi_{\lambda}(t, \omega, x) d x d \omega d t,
\end{aligned}
$$




$$
\begin{aligned}
J_{4}(\lambda, r, s):= & \int_{0}^{T} \int_{Q} \eta(x)\left[\int_{\mathbb{S}} h\left(\omega^{\prime}, \omega\right) R_{1, \lambda, s}\left(t, \omega^{\prime}, x\right) d \omega^{\prime}\right] \\
& R_{2, \lambda, r}^{*}(t, \omega, x) d x d \omega d t .
\end{aligned}
$$

Taking the limit as $r \rightarrow 1^{-}$in the above expressions, we get from the definition of $\chi_{r}, J_{i}(\lambda, r, s) \rightarrow J_{i}(\lambda, s)$, where

$$
\begin{aligned}
& J_{1}(\lambda, s):=\int_{0}^{T} \int_{\Omega} \eta(x)\left[\int_{\mathbb{S}} h\left(\omega^{\prime}, \widetilde{\omega}\right) \chi_{s}\left(\widetilde{\omega}, \omega^{\prime}\right) \Phi_{\lambda}\left(t, \omega^{\prime}, x\right) d \omega^{\prime}\right] \Psi_{\lambda}(t, \widetilde{\omega}, x) d x d t, \\
& J_{2}(\lambda, s):=\int_{0}^{T} \int_{Q} \eta(x)\left[\int_{\mathbb{S}} h\left(\omega^{\prime}, \omega\right) \chi_{s}\left(\widetilde{\omega}, \omega^{\prime}\right) \Phi_{\lambda}\left(t, \omega^{\prime}, x\right) d \omega^{\prime}\right] S_{2, \lambda}^{*}(t, \omega, x) d x d \omega d t, \\
& J_{3}(\lambda, s):=\int_{0}^{T} \int_{\Omega} \eta(x)\left[\int_{\mathbb{S}} h\left(\omega^{\prime}, \widetilde{\omega}\right) R_{1, \lambda, s}\left(t, \omega^{\prime}, x\right) d \omega^{\prime}\right] \Psi_{\lambda}(t, \widetilde{\omega}, x) d x d t, \\
& J_{4}(\lambda, s):=\int_{0}^{T} \int_{Q} \eta(x)\left[\int_{\mathbb{S}} h\left(\omega^{\prime}, \omega\right) R_{1, \lambda, s}\left(t, \omega^{\prime}, x\right) d \omega^{\prime}\right] S_{2, \lambda}^{*}(t, \omega, x) d x d \omega d t
\end{aligned}
$$

and $S_{2, \lambda}^{*}$ is the unique solution of

$$
\left\{\begin{array}{l}
\partial_{t} S+\omega \cdot \nabla S-q S=-q K_{\kappa_{2}}^{*}[S]+\mathrm{e}^{-\mathrm{i} \lambda t} q Z_{2, \lambda}^{*}, \\
S(T, \omega, x)=0, \quad(\omega, x) \in \mathbb{S} \times \Omega \\
S(T, \omega, \sigma)=0, \quad(\omega, \sigma) \in \Sigma^{+}
\end{array}\right.
$$

Moreover, from (4.5) and (4.2), it follows that $L(\lambda, r, s) \rightarrow L(\lambda, s)$, where

$$
\begin{aligned}
L(\lambda, s):= & \int_{0}^{T} \int_{\partial \Omega}(\widetilde{\omega} \cdot v(\sigma))^{+}\left(\widetilde{\mathcal{A}}_{1}\left[f_{\lambda, s}\right]-\widetilde{\mathcal{A}}_{2}\left[f_{\lambda, s}\right]\right) \\
& (t, \widetilde{\omega}, \sigma) \Psi_{\lambda}(t, \widetilde{\omega}, \sigma) d \sigma d t \\
= & \int_{0}^{T} \int_{\partial \Omega}(\widetilde{\omega} \cdot v(\sigma))^{+}\left(R_{1, \lambda, s}(t, \widetilde{\omega}, \sigma)-R_{2, \lambda, s}(t, \widetilde{\omega}, \sigma)\right) \\
& \Psi_{\lambda}(t, \widetilde{\omega}, \sigma) d \sigma d t
\end{aligned}
$$

where $\widetilde{\mathcal{A}}_{i}\left[f_{\lambda, s}\right]$ denotes the zero extension of $\mathcal{A}_{i}\left[f_{\lambda, s}\right]$ on $\partial \Omega$. Therefore, by taking the limit as $r \rightarrow 1^{-}$in (4.4), we have

$$
J_{1}(\lambda, s)+J_{2}(\lambda, s)+J_{3}(\lambda, s)+J_{4}(\lambda, s)=L(\lambda, s) .
$$


Now, it is time to take the limit as $s \rightarrow 1^{-}$. For the first two terms of the right hand side of the above identity, we get (for $i=1,2) J_{i}(\lambda, s) \rightarrow J_{i}(\lambda)$, where

$$
\begin{aligned}
J_{1}(\lambda): & =\int_{0}^{T} \int_{\Omega} \eta(x) h(\widetilde{\omega}, \widetilde{\omega}) \Phi_{\lambda}(t, \widetilde{\omega}, x) \Psi_{\lambda}(t, \widetilde{\omega}, x) d x d t \\
& =h(\widetilde{\omega}, \widetilde{\omega}) \int_{0}^{T} \int_{\Omega} \eta(x) \phi(x-t \widetilde{\omega})^{2} d x d t, \\
J_{2}(\lambda): & =\int_{0}^{T} \int_{Q} \eta(x) h(\widetilde{\omega}, \omega) \Phi_{\lambda}(t, \widetilde{\omega}, x) S_{2, \lambda}^{*}(t, \omega, x) d x d \omega d t .
\end{aligned}
$$

On the other hand, the dependence on $s$ in the other terms is given by $R_{1, \lambda, s}$ and $R_{2, \lambda, s}$, which are the solution of $(j=1,2)$

$$
\left\{\begin{array}{l}
\partial_{t} R+\omega \cdot \nabla R+q R=q K_{\kappa_{j}}[R]+\mathrm{e}^{\mathrm{i} \lambda t} q Z_{j, \lambda, s}, \\
R(0, \omega, x)=0, \quad(\omega, x) \in \mathbb{S} \times \Omega, \\
R(t, \omega, \sigma)=0, \quad(\omega, \sigma) \in \Sigma^{+},
\end{array}\right.
$$

where

$$
Z_{j, \lambda, s}(t, \omega, x):=\int_{\mathbb{S}} \kappa_{j}\left(x, \omega^{\prime}, \omega\right) \chi_{s}\left(\widetilde{\omega}, \omega^{\prime}\right) \Phi_{\lambda}\left(t, \omega^{\prime}, x\right) d \omega^{\prime} .
$$

It is an immediate consequence of (4.2) and the Lebesgue's Theorem that, as $s \rightarrow 1, Z_{j, \lambda, s} \rightarrow Z_{j, \lambda}$ in $C\left([0, T] ; L^{2}(Q)\right)$, where

$$
Z_{j, \lambda}(t, \omega, x):=\kappa_{j}(x, \widetilde{\omega}, \omega) \Phi_{\lambda}(t, \widetilde{\omega}, x) .
$$

Hence,

$$
\lim _{s \rightarrow 1^{-}} R_{j, \lambda, s}=S_{j, \lambda} \quad \text { in } \quad C\left([0, T] ; L^{2}(Q)\right),
$$

where $S_{j, \lambda}$ is the solution of

$$
\left\{\begin{array}{l}
\partial_{t} S+\omega \cdot \nabla S+q S=q K_{\kappa_{j}}[S]+\mathrm{e}^{\mathrm{i} \lambda t} q Z_{j, \lambda}, \\
S(0, \omega, x)=0, \quad(\omega, x) \in \mathbb{S} \times \Omega, \\
S(t, \omega, \sigma)=0, \quad(\omega, \sigma) \in \Sigma^{-},
\end{array}\right.
$$

and

$$
Z_{j, \lambda}(t, \omega, x):=c_{j}(x) h(\widetilde{\omega}, \omega) \Phi_{\lambda}(t, \widetilde{\omega}, x) .
$$


Therefore, $J_{i}(\lambda, s) \rightarrow J_{i}(\lambda),(i=3,4)$ and $L(\lambda, s) \rightarrow L(\lambda)$, where

$$
\begin{aligned}
& J_{3}(\lambda):=\int_{0}^{T} \int_{\Omega} \eta(x)\left[\int_{\mathbb{S}} h\left(\omega^{\prime}, \widetilde{\omega}\right) S_{1, \lambda}\left(t, \omega^{\prime}, x\right) d \omega^{\prime}\right] \Psi_{\lambda}(t, \widetilde{\omega}, x) d x d t, \\
& J_{4}(\lambda):=\int_{0}^{T} \int_{Q} \eta(x)\left[\int_{\mathbb{S}} h\left(\omega^{\prime}, \widetilde{\omega}\right) S_{1, \lambda}\left(t, \omega^{\prime}, x\right) d \omega^{\prime}\right] S_{2, \lambda}^{*}(t, \omega, x) d x d \omega d t . \\
& L(\lambda):=\int_{0}^{T} \int_{\partial \Omega}(\widetilde{\omega} \cdot v(\sigma))^{+}\left(S_{1, \lambda}(t, \widetilde{\omega}, \sigma)-S_{2, \lambda}(t, \widetilde{\omega}, \sigma)\right) \Psi_{\lambda}(t, \widetilde{\omega}, \sigma) d \sigma d t
\end{aligned}
$$

and we obtain

$$
\left|J_{1}(\lambda)\right| \leq\left|J_{2}(\lambda)\right|+\left|J_{3}(\lambda)\right|+\left|J_{4}(\lambda)\right|+|L(\lambda)|,
$$

where

$$
\begin{aligned}
\left|J_{2}(\lambda)\right| \leq & \|\eta\|_{\infty}\|h\|_{\infty} \mathrm{e}^{M T} \int_{0}^{T} \int_{Q}\left|\phi(x-t \widetilde{\omega}) S_{2, \lambda}^{*}(t, \omega, x)\right| d x d \omega d t, \\
\left|J_{3}(\lambda)\right| \leq & \|\eta\|_{\infty}\|\phi\|_{\infty} \mathrm{e}^{M T}\left\|K_{h}\left[S_{1, \lambda}\right]\right\|_{L^{2}\left(0, T ; L^{2}(Q)\right)}, \\
\left|J_{4}(\lambda)\right| \leq & \|\eta\|_{\infty}\left\|K_{h}\left[S_{1, \lambda}\right]\right\|_{L^{2}\left(0, T ; L^{2}(Q)\right)}\left\|S_{2, \lambda}^{*}\right\|_{L^{2}\left(0, T ; L^{2}(Q)\right)}, \\
|L(\lambda)| \leq & \|\phi\|_{\infty} \mathrm{e}^{M T} \int_{0}^{T} \int_{\partial \Omega}(\widetilde{\omega} \cdot v(\sigma))^{+} \\
& \left|S_{1, \lambda}(t, \widetilde{\omega}, \sigma)-S_{2, \lambda}(t, \widetilde{\omega}, \sigma)\right| d \sigma d t .
\end{aligned}
$$

Since $\phi \in C_{0}^{\infty}\left(\Omega_{\varepsilon}\right)$, it follows from the choice of $\varepsilon$ that the function $(t, \omega, x) \mapsto$ $\phi(x-t \tilde{\omega})$ belongs to $H_{0}^{1}\left(0, T ; L^{2}(Q)\right)$ (as a constant function on $\left.\omega\right)$. Hence, we have

$$
\left|J_{2}(\lambda)\right| \leq\|\rho\|_{\infty} \mathrm{e}^{M T}\|\phi\|_{H_{0}^{1}\left(0, T ; L^{2}(Q)\right)}\left\|S_{2, \lambda}^{*}\right\|_{H^{-1}\left(0, T ; L^{2}(Q)\right)} .
$$

On the other hand, from the weak convergence to zero in $L^{2}\left(0, T ; L^{2}(Q)\right)$ of $S_{1, \lambda}$, it follows that

$$
\lim _{\lambda \rightarrow+\infty}\left\|K_{h}\left[S_{1, \lambda}\right]\right\|_{L^{2}\left(0, T ; L^{2}(Q)\right)}=0 .
$$


Hence, we have from (4.15)-(4.17) and Lemma 3.3,

$$
\begin{aligned}
\left|J_{1}(\lambda)\right|= & |h(\widetilde{\omega}, \widetilde{\omega})|\left|\int_{0}^{T} \int_{\Omega} \eta(x) \phi(x-t \widetilde{\omega})^{2} d x d t\right| \\
\leq & C(\lambda)\|\eta\|_{\infty}+C_{2} \int_{0}^{T} \int_{\partial \Omega}(\widetilde{\omega} \cdot v(\sigma))^{+} \\
& \left|S_{1, \lambda}(t, \widetilde{\omega}, \sigma)-S_{2, \lambda}(t, \widetilde{\omega}, \sigma)\right| d \sigma d t,
\end{aligned}
$$

where $C(\lambda) \rightarrow 0$ as $\lambda \rightarrow+\infty$.

Since $(\operatorname{supp} \phi+s \widetilde{\omega}) \cap \Omega=\emptyset$ for all $|s| \geq T$, we have

$$
\begin{aligned}
\left|\int_{0}^{T} \int_{\Omega} \eta(x) \phi(x-t \widetilde{\omega})^{2} d x d t\right| & =\left|\int_{\mathbb{R}^{N}} \int_{0}^{T} \eta(y+s \widetilde{\omega}) \phi(y)^{2} d s d y\right| \\
& =\left|\int_{\mathbb{R}^{N}} \int_{-\infty}^{\infty} \eta(y+s \widetilde{\omega}) \phi(y)^{2} d s d y\right| \\
& =\left|\int_{\mathbb{R}^{N}} P_{\tilde{\omega}}[\eta](y) \phi(y)^{2} d y\right|
\end{aligned}
$$

and we get

$$
\begin{gathered}
|h(\widetilde{\omega}, \widetilde{\omega})|\left|\int_{\mathbb{R}^{N}} P_{\tilde{\omega}}[\eta](y) \phi(y)^{2} d y\right| \leq C(\lambda)\|\eta\|_{\infty} \\
+C_{2} \int_{0}^{T} \int_{\partial \Omega}(\widetilde{\omega} \cdot v(\sigma))^{+}\left|S_{1, \lambda}(t, \widetilde{\omega}, \sigma)-S_{2, \lambda}(t, \widetilde{\omega}, \sigma)\right| d \sigma d t
\end{gathered}
$$

We are now in position to conclude the proof. First of all, we consider in the above inequality the directions $\widetilde{\omega}_{1}, \ldots, \widetilde{\omega}_{k}$ and the functions $\phi_{1}, \ldots, \phi_{k}$ given by Lemma 4.1, in such a way that we can write

$$
\begin{gathered}
C_{0}\left\|c_{1}-c_{2}\right\|_{\infty} \leq C(\lambda)\left\|c_{1}-c_{2}\right\|_{\infty}+ \\
+C_{2} \sum_{j=1}^{k} \int_{0}^{T} \int_{\partial \Omega}\left(\widetilde{\omega}_{j} \cdot v(\sigma)\right)^{+}\left|S_{1, \lambda}\left(t, \widetilde{\omega}_{j}, \sigma\right)-S_{2, \lambda}\left(t, \widetilde{\omega}_{j}, \sigma\right)\right| d \sigma d t
\end{gathered}
$$

for some constant $C_{0}>0$. If we denote by $u_{i, j}(t, \omega, \sigma)=\chi_{s}\left(\widetilde{\omega}_{j}, \omega\right) \Phi_{\lambda}(t, \omega, x)+R_{i, \lambda, s}(t, \omega, x), \quad i=1,2, j=1, \ldots, k$ 
it follows from (4.2) that, as $s \rightarrow 1^{-}, u_{i, j} \rightarrow u_{i, j}^{\#}$, where

$$
u_{i, j}^{\#}=\delta_{\tilde{\omega}_{j}} \Phi_{\lambda}+S_{i, \lambda}, \quad i=1,2, \quad j=1, \ldots, k
$$

and $\delta_{\tilde{\omega}_{j}}$ is the spherical atomic measure concentrated on $\widetilde{\omega}_{j}$.

It is clear from (4.13) that $u_{1, j}^{\#}(t, \omega, \sigma)=u_{2, j}^{\#}(t, \omega, \sigma)$, for $\sigma \in \Sigma_{\omega}^{-}$and $j=1, \ldots, k$. Moreover, $u_{1, j}^{\#}-u_{2, j}^{\#}=S_{1, \lambda}-S_{2, \lambda}$. Therefore, if $u_{1, j}^{\#}\left(t, \widetilde{\omega}_{j}, \sigma\right)=$ $u_{2, j}^{\#}\left(t, \widetilde{\omega}_{j}, \sigma\right)$ on $\Sigma_{\tilde{\omega}_{j}}^{+}$, for $j=1, \ldots, k$, it follows that

$$
C_{0}\left\|c_{1}-c_{2}\right\|_{\infty} \leq C(\lambda)\left\|c_{1}-c_{2}\right\|_{\infty}
$$

and the conclusion follows easily if we choose $\lambda>0$ large enough.

\section{REFERENCES}

[1] A.P. Calderón, On an inverse boundary value problem. Seminars on Numerical Analysis and Application to Continuum Physics, SBM (Rio de Janeiro 1980), 65-73.

[2] M. Cessenat, Théorèmes de trace $L^{p}$ pour des espaces de fonctions de la neutronique. C.R. Acad. Sci. Paris, Série I, 299 (1984), 831-834.

[3] M. Cessenat, Théorèmes de trace pour des espaces de fonctions de la neutronique. C.R. Acad. Sci. Paris, Série I, 300 (1985), 89-92.

[4] M. Choulli and P. Stefanov, Inverse scattering and inverse boundary value problem for the linear Boltzmann equation. Comm. Part. Diff. Equations, 21(5-6) (1996), 763-785.

[5] R. Cipolatti, C.M. Motta and N.C. Roberty, Stability Estimates for an Inverse Problem for the Linear Boltzmann Equation. Revista Matemática Complutense, 19(1) (2006), 113-132.

[6] R. Cipolatti and Ivo F. Lopez, Determination of coefficients for a dissipative wave equation via boundary measurements. J. Math. Anal. Appl., 306 (2005), 317-329.

[7] R. Dautray and J.-L. Lions, Mathematical Analysis and Numerical Methods for Science and Technology, 6 (1993), Springer-Verlag.

[8] G.B. Folland, Introduction to Partial Differential Equations. Mathematical Notes, 17 (1976), Princeton University Press.

[9] M. Mokhtar-Kharroubi, Mathematical Topics in Neutron Transport Theory - New Aspects; Series on Advances in Mathematics for Applied Sciences, 46 (1997), World Scientific.

[10] M. Reed and B. Simon, Methods of Modern Physics, 3 (1993), Springer-Verlag.

[11] V.G. Romanov, Estimation of stability in the problem of determining the attenuation coefficient and the scattering indicatrix for the transport equation. Sibirsk. Mat. Zh., 37(2) (1996), 361-377, iii; translation in Siberian Math. J., 37(2) (1996), 308-324. 
[12] V.G. Romanov, Stability estimate in the three-domensional inverse problem for the transport equation. J. Inverse Ill-Posed Probl., 5(5) (1997), 463-475.

[13] P. Stefanov and G. Uhlmann, Optical tomography in two dimensions. Methods and Applications of Analysis, 10 (2002), 1-9.

[14] A. Tamasan, An inverse boundary value problem in two dimensional transport. Inverse Problems, 18 (2002), 209-219.

[15] J.N. Wang, Stability estimates of an inverse problem for the stationary transport equation. Ann. Inst. Henri Poincaré, 70(5) (1999), 473-495. 